EUDET-Report-2008-01

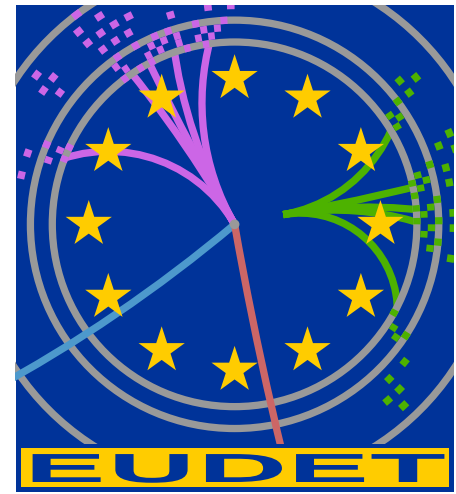

\title{
The EUDET High Resolution Pixel Telescope*
}

\author{
Philipp Roloff ${ }^{\dagger}$
}

October 14, 2008

\begin{abstract}
Within the EUDET consortium a high resolution pixel beam telescope is developed. The telescope consists of up to six planes of monolithic active pixel sensors and can be operated in a magnetic field of $1.2 \mathrm{~T}$. A flexible data acquisition is available for the telescope and the system is equipped with all required infrastructure. Since the first installation of a demonstrator telescope in 2007, it has been extensively tested and used by various detector R\&D groups. Results from testbeam measurements to obtain the telescope performance are described here. The sensors of the final telescope will provide digital outputs and zero suppression.
\end{abstract}

*Presentation at the 8th International Conference on Position Sensitive Detectors (PSD08) in Glasgow, September 2008

${ }^{\dagger}$ DESY, Hamburg, Germany 


\section{Introduction}

A linear electron-positron collider operated a the centre-of-mass energy of at least $500 \mathrm{GeV}$ is the next great international project in high energy physics. The EUDET project which is supported by the EU in the 6th Framework Programme (FP6) aims to provide infrastructures for the $\mathrm{R} \& \mathrm{D}$ of detector technologies towards the international linear collider. Within the EUDET project the JRA1 activity works on the improvement of testbeam infrastructures. For this purpose a high resolution pixel telescope is developed. The design goals include a high position resolution $(\sigma<3.0 \mu \mathrm{m})$ and readout rate of $1 \mathrm{kHz}$. Additionally, the telescope can be operated in a $1.2 \mathrm{~T}$ solenoid magnet (PCMAG).

The construction of the telescope is performed in two steps. In June 2007 the so-called demonstrator telescope was installed for the first time where an analog readout is used. After the first successful operation at the electron beam at DESY, the demonstrator was transported to CERN and the performance was studied using $180 \mathrm{GeV}$ hadrons at the SPS [1]. After the first successful integration of a Device Under Test (DUT) in September 2007 [2], the demonstrator telescope has been used by various groups and was improved continuously. More data processing will be moved to the sensors in the fully digital final telescope which will become available in spring 2009 .

\section{The sensor}

The MimoTEL sensor used for the demonstrator telescope was developed by the CNRSIHPC institute in Strasbourg, France. Is is a Monolithic Active Pixel Sensor (MAPS) produced in the AMS 0.35 OPTO process. Sensors with a thickness of the epitaxial layer of 14 or $20 \mu \mathrm{m}$ are available. In total the sensor is $680 \mu \mathrm{m}$ thick. Four sub-arrays of $64 \times 256$ pixels are read in parallel. With a pixel pitch of $30 \times 30 \mu \mathrm{m}^{2}$ this results in a sensor size of $7.7 \times 7.7 \mathrm{~cm}^{2}$. A high resolution sensor with a pitch of $10 \mu \mathrm{m}$ can be located close to the DUT to increase the resolution further. The effects of different telescope geometries and different beam energies have been studied using simulations in great detail $[3,4]$. The sensor for the final telescope will be described in section 7 .

\section{The DAQ system}

The DAQ system can be summarised as follows: All data from the sensors is transferred via frontend boards to an intermediate readout and data reduction board called EUDRB (EUDET Data Reduction Board) [5]. The EUDRB board allows to perform the first steps of the data processing online. Two I/O busses are supported: For the telescope the VME64x bus is used to allow high speed data transfer and synchronous operation with other devices while an USB2.0 interface is foreseen for standalone testing. A mother / daughter board scheme has been followed to maximise the flexibility. All computing and memory elements are located on the motherboard while the sensor specific components 
have been implemented on removable and interchangeable daughter cards. The two following modes of operation have been implemented in the EUDRB firmware so far:

- Transparent mode: All pixel signals are transferred without further data processing. This mode is important for debugging and for the characterisation of the telescope sensors itself.

- Zero suppressed mode (ZS): The correlated double sampling (CDS) is performed online and only the signals and addresses of pixels above a certain userdefined threshold are transferred. This mode is intended for datataking at high rates keeping the output files reasonably small.

The output of the EUDRB boards is collected by a MVME6100 single board computer which is located in the same VME64x crate. Finally, the data are send to the main DAQ PC using gigabit ethernet. This computer can also collect the information from the device under test (DUT).

Another important component of the DAQ system is the trigger logic unit (TLU) [6]. It is considered as the replacement of a NIM crate and can generate any coincidence or anticoincidence of four trigger scintillators. Six LVDS and two TTL interfaces are provided. Furthermore, the TLU generates event numbers and time stamps. It is connected by USB2.0 to a control PC running the Linux operating system that is in turn connected to the main DAQ PC through gigabit ethernet.

Figure 1 shows the DAQ system of the EUDET pixel telescope as described above.

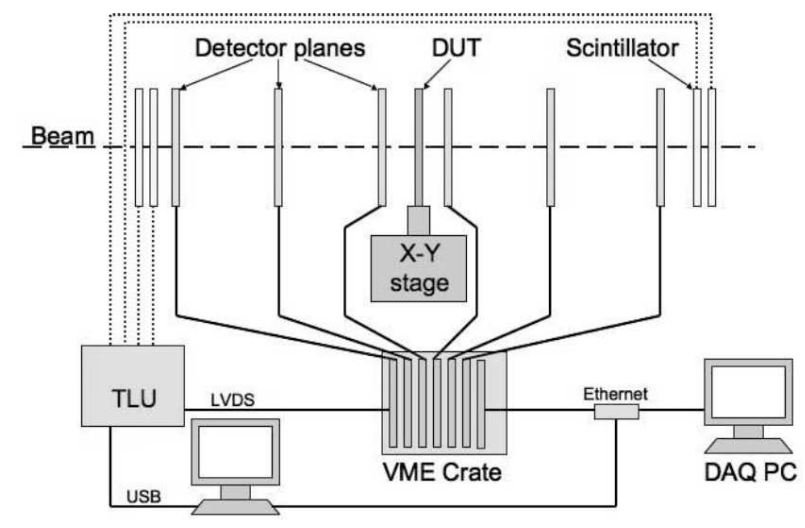

Figure 1: The DAQ system of the EUDET pixel telescope.

A custom DAQ system named EUDAQ has been implemented in $\mathrm{C}++$ [8]. Several producer tasks communicate with a global run control using sockets. These producer tasks connect to the hardware of the beam telescope, to the TLU and eventually to the DUT. Data from all producers is sent to the central data collector and can be monitored by several processes. An online monitor based on the ROOT framework showing online data quality monitoring histograms as well as a process to collect log messages are available. EUDAQ runs on MacOS, Linux and Windows using cygwin. 


\section{User integration}

Different scenarios for the integration of the DUT in the DAQ system of the EUDET pixel telescope are possible:

- Integration at hardware level: In this case the user has to provide a hardware interface able to read out the telescope sensors and the DUT. This approach is supported by the EUDRB boards, but is only feasible for some dedicated DUTs.

- Integration at DAQ software level: The user provides own DAQ hardware to read out the DUT, but the data are be treated by a common DAQ software. In case EUDAQ is used as the common DAQ system, a producer to read out the DUT needs to be implemented.

- Integration at trigger level: This default scenario was chosen by most users so far, because it is easy to implement and relatively safe. Different hardware and software are used for the telescope and the DUT. The synchronisation is done using the trigger, busy and reset logic provided by the TLU. To protect against slippage of event numbers between the telescope and the DUT, the event number provided by the TLU can be read by the DUT.

- Integration at data level: Both, the DUT and the telescope use their own dedicated DAQ hardware and software. The data streams are combined offline by inter process communication. In this scenario the synchronisation and the configuration during the start-up might be difficult.

\section{The offline analysis software}

For the offline reconstruction of track positions in the DUT the software package EUTelescope has been developed which is implemented as a set of Marlin processors [9]. This design allows to integrate the DUT data at different steps of the analysis chain. Furthermore, the package is prepared to be executed on the Grid to allow a fast processing of large datasets.

Figure 2 summarises the structure of the offline analysis package. Each step in the analysis procedure is implemented in a separate Marlin processor. It is possible to run each processor separately or to execute the whole analysis chain by a single command. In the first step the data is converted from a native format used by the EUDAQ software to the LCIO format. Afterwards a pedestal correction is applied and clusters are searched for. It is possible to improve the reconstructed cluster positions using the $\eta$ algorithm. Clusters are accordingly transformed into hits in the telescope frame of reference. The alignment procedure is based on the Millepede II package and uses full tracks in a simultaneous fit to derive the alignment parameters. Finally, tracks are are fitted using the hits after the alignment constants have been applied. The result of the track fit can be saved in a ROOT file if needed by the user of the telescope. 


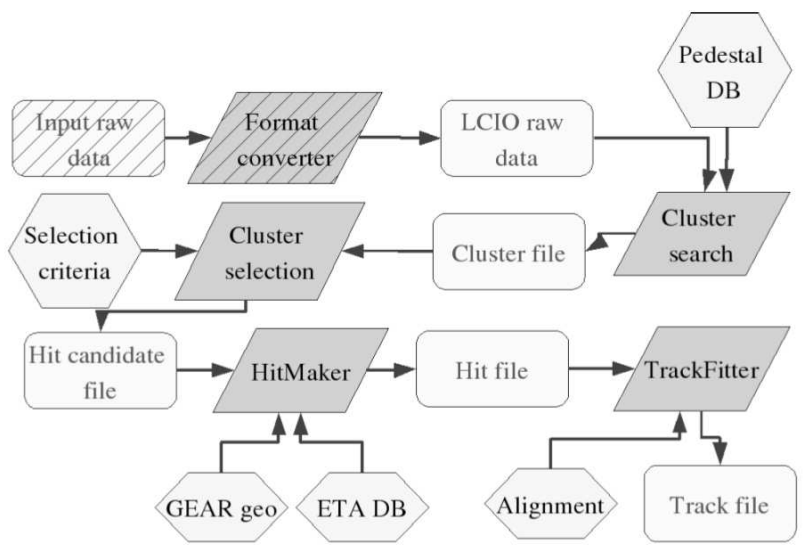

Figure 2: Schematic overview of the offline analysis package EUTelescope.

\section{Testbeam results}

In this section results from testbeam measurements to characterise the demonstrator telescope are discussed. The first part of the data was taken at DESY in August 2007 using a compact setup of the telescope where 5 MimoTELs were mounted in a distance of $2.5 \mathrm{~cm}$. Further studies were performed in September 2007 at CERN. Here the sensors were mounted in two separate boxes with a gap of $34 \mathrm{~cm}$ for a DUT in the middle. The first box contained 3 sensors while only 2 sensors were located in the second box. Within the boxes, the sensors were mounted in a distance of $10 \mathrm{~cm}$.

First, the telescope sensors were characterised with minimum ionising particles. In the setup at CERN two different types of MimoTEL sensors have been used, one kind with a $14 \mu \mathrm{m}$ thick epitaxial layer and another version had $20 \mu \mathrm{m}$ thick epitaxial layers. Table 1 shows the signals in ADC counts and the signal to noise ratios for seed pixels and for clusters consisting of $3 \times 3$ pixels. The given values represent the most probable value of Laudau fits. During the data was taken the telescope was operated using only moderate cooling keeping the sensors at a constant temperature between 20 and 22 degrees Celsius.

\begin{tabular}{|c|c|cc|cc|}
\hline Sensor & Epi thickness & Seed pixel & $3 \times 3$ Cluster & \\
& & ADC & S/N & ADC & S/N \\
\hline \hline 0 & $14 \mu \mathrm{m}$ & 47.2 & 12.5 & 131.0 & 11.2 \\
1 & & 46.2 & 12.2 & 129.0 & 10.9 \\
2 & & 47.3 & 12.8 & 130.3 & 11.3 \\
\hline 3 & $20 \mu \mathrm{m}$ & 47.5 & 10.9 & 151.4 & 11.0 \\
4 & & 46.3 & 12.6 & 147.6 & 13.2 \\
\hline
\end{tabular}

Table 1: Signals in ADC counts and signal to noise ratios for seed pixels and $3 \times 3$ clusters in the individual planes measured with the setup used at CERN in September 2007.

For both types of sensors the same amount of charge is collected in the seed pixels. The 
sensor 3 shows a lower signal to noise ratio compared to the other sensors. This is caused by the fact that sensor 3 has a $20 \%$ larger mean single pixel noise which was introduced by the used acquisition and sampling electronics.

In the sensors with thicker epitaxial layers more charge is collected in clusters of $3 \times 3$ pixels. This indicates a greater signal production but also a larger charge spread in these sensors compared to the sensors with a $14 \mu \mathrm{m}$ epitaxial layer. For both sensor types the mean cluster size is around 8 pixels if a threshold of 2.5 times the pixel noise is applied. Before tracks can be fitted, the telescope planes need to be aligned. Typically the sensors are shifted by a few hundred $\mu \mathrm{m}$ in the directions perpendicular to the beam and rotated a few mrad around the beam direction. As an example table 2 illustrates the result of the alignment procedure for data taken using a beam of $3 \mathrm{GeV}$ electrons at DESY. Tracks were fitted using the constants provided by the alignment procedure. The mean values of Gaussian functions fitted to the observed residuals in the individual planes are shown. For a perfectly aligned telescope these mean values should vanish. The results demonstrate that the alignment of the telescope is possible with sufficient precision for the intended applications.

\begin{tabular}{|c|c|c|}
\hline Sensor & Mean X $[\mu \mathrm{m}]$ & Mean Y $[\mu \mathrm{m}]$ \\
\hline \hline 0 & $-0.003 \pm 0.002$ & $-0.023 \pm 0.002$ \\
1 & $-0.012 \pm 0.004$ & $0.036 \pm 0.005$ \\
2 & $0.032 \pm 0.004$ & $0.005 \pm 0.005$ \\
3 & $-0.020 \pm 0.004$ & $-0.005 \pm 0.005$ \\
4 & $0.001 \pm 0.002$ & $-0.002 \pm 0.002$ \\
\hline
\end{tabular}

Table 2: Mean values of the residual distributions for tracks fitted after the alignment.

If multiple scattering can be neglected, it is possible to fit linear tracks. This is the case for the data recorded using a beam of $180 \mathrm{GeV}$ hadrons in September 2007 at CERN. The $X$ an $Y$ coordinates were fitted separately. The resulting track candidates were required fulfill a cut on $\chi^{2}<20$ in both directions. More than one track candidate per event was allowed. Figure 3 shows the residuals of the tracks in the middle out of 5 sensor planes. Here the middle telescope sensor acts as DUT while the other planes are used to predict the track positions in the DUT. The observed width is consistent with the expectation for the given telescope geometry assuming a position resolution of $3.0 \mu \mathrm{m}$ for the DUT as well as for the other sensors used to fit tracks. Also measurements at DESY using 3 and $6 \mathrm{GeV}$ electrons based on an extrapolation to infinite energy are in agreement with this sensor resolution.

If the influence of the telescope material can not be neglected, the result can be improved taking the contribution from multiple scattering into account in an analytical fitting approach [4]. Results are shown in figure 4. The widths of the residual distributions measured in the 5 telescope sensors are shown. The actual plane was excluded from the track fit. Results for beams of 3 and $6 \mathrm{GeV}$ electrons are in agreement with the expectation and with a simulation based on Geant4. In both cases a position resolution of the sensors of $3.0 \mu \mathrm{m}$ was assumed. This demonstrates that a good telescope performance 
can be achieved even at beam energies of a few $\mathrm{GeV}$.

During the summer 2008 the full demonstrator consisting of 6 sensors running in zero suppressed mode was operated at CERN. The analysis of the data from these measurements is ongoing.

\section{Outlook: Towards the digital readout}

The main difference between the demonstrator and the final telescope which will be assembled and tested starting in spring 2009 is the new sensor called Mimosa 26. This chip consists of 1152 columns of 576 pixels. With a pitch of $18.4 \mu \mathrm{m}$ the Mimosa 26 has an active area of $21.2 \times 10.6 \mathrm{~cm}^{2}$, which is a factor of 3.8 larger than the MimoTEL of the demonstrator telescope. The integration time of about $110 \mu$ s allows to read out about $10^{4}$ frames per second. The sensor provides binary output and is equipped with a zero suppression micro-circuit. To reduce the influence of multiple scattering, the Mimosa 26 will be thinned.

\section{Summary}

The demonstrator telescope is running successfully during several testbeam measurements since about one year. Modularity was one of the most important design aspects for the DAQ hardware and software as well as for the offline analysis package. The analysis of testbeam data shows that the performance of the demonstrator fulfills the expectations. An increased active area and zero suppression on the sensors will be offered by the final telescope. Interested groups are welcome to contact the EUDET consortium for the exploitation of the device.
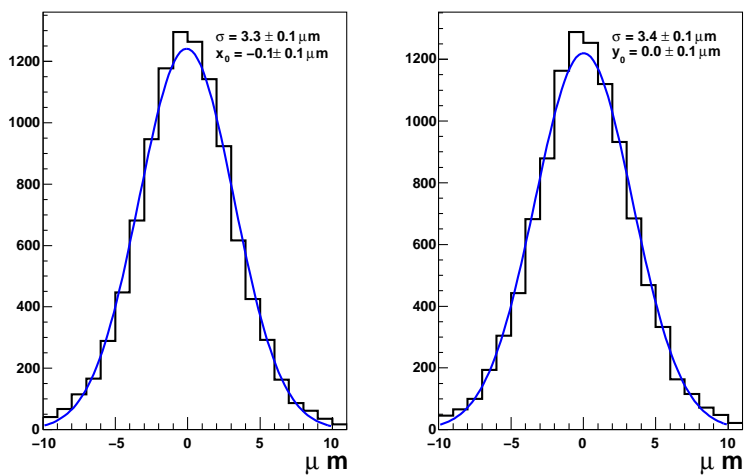

Figure 3: Residuals in the middle telescope sensor in the $X$ (left) and $Y$ (right) directions. This sensor was excluded from the track fit and hence acts as DUT. 


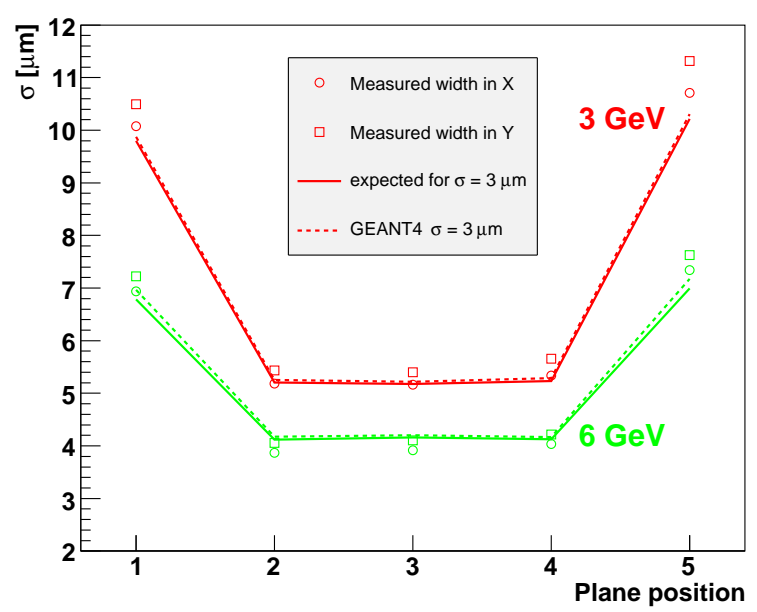

Figure 4: Widths of the residuals in different telescope planes.

\section{Acknowledgement}

This work is supported by the Commission of the European Communities under the $6^{\text {th }}$ Framework Programme "Structuring the European Research Area", contract number RII3-026126.

\section{References}

[1] A. Bulgheroni, EUDET-Report-2007-06 (2007).

[2] L. Reuen, EUDET-Memo-2007-40 (2007).

[3] T. Klimkovich, EUDET-Memo-2007-06 (2007).

[4] A.F. Zarnecki and P. Niezurawski, EUDET-Memo-2007-01, arXiv:physics/0703058v1 (2007).

[5] A. Cotta Ramusino, EUDET-Memo-2007-36 (2007).

[6] D. Cussans, EUDET-Memo-2007-02 (2007).

[7] D. Haas, Proc. of the LCWS'07, Hamburg, EUDET-Report-2007-08 (2007).

[8] E. Corrin, D. Haas and M. Pohl, EUDET-Memo-2006-07 (2006).

[9] A. Bulgheroni, T. Klimkovich, P. Roloff and A.F. Zarnecki, EUDET-Memo-2007-20 (2007). 